\title{
Planning and Evaluation of Reorienting Osteotomies of the Proximal Femur in Cases of SCFE Using Virtual Three-Dimensional Models
}

\author{
Jens A. Richolt ${ }^{1}$, Matthias Teschner ${ }^{2}$, Peter Everett ${ }^{1}$, Bernd Girod ${ }^{2}$, \\ Michael B. Millis ${ }^{3}$, and Ron Kikinis ${ }^{1}$ \\ ${ }^{1}$ Surgical Planning Laboratory, Brigham and Women's Hospital, Harvard Medical School, \\ 75, Francis Street, Boston, MA, 02115, USA \\ \{jens, peverett, kikinis\}@bwh.harvard.edu \\ http://splweb.bwh.harvard.edu:8000/pages/index.html \\ ${ }^{2}$ Telecommunications Institute, University Erlangen-Nuremberg, \\ Cauer Strasse 7, D-91058 Erlangen, Germany \\ ${ }^{3}$ Children's Hospital, Orthopaedic Surgery, Harvard Medical School, \\ 300, Longwood Avenue, Boston, MA, 02115, USA
}

\begin{abstract}
Slipped Capital Femoral Epiphysis (SCFE) is a disease affecting the geometry of adolescent hips. Evaluation of the slippage as well planning of correction surgeries is a major three-dimensional problem. Therefore, the current clinical approach, which is based on biplanar plain radiographs, is not satisfying.

We have developed a software environment for planning and evaluation of reorienting osteotomies in severe cases of SCFE. In our system CT-based virtual surface models fitted by oriented bounding boxes (OBB) are manipulated. The hip motion as well as a correction surgery can be simulated. Both are controlled by collision detection. Therefore, the motion is based on the surface geometry of the joint partners rather than on a predefined, fixed rotation center. The pre- and simulated postoperative evaluation uses the range of motion as the essential parameter. The surgeon can obtain valuable information about the geometric correlation of the physiologic joint faces along hip motion.
\end{abstract}

\section{Introduction}

Slipped Capital Femoral Epiphysis (SCFE) is a condition affecting the proximal femur of adolescents. The SCFE is defined as the slippage of the femoral head on the proximal growth plate against the femoral neck. Usually this displacement occurs in a posterior and inferior direction (Fig. 1). In severe cases these events can determine a significant loss of hip function. Initially the loss of motion may be well tolerated, but eventually pain and stiffness of the joint will result from arthritic degeneration [2].

The standard procedure (so called in-situ-pinning) in SCFE aims towards the prevention of further slippage, which does not affect the post slippage geometry. In severe cases an additional correction osteotomy might be considered to improve the range of motion and the containment of femoral head and acetabulum [6,13]. The central part of this surgery consists of a cut through the proximal femur and a reorientation of the proximal part. The most common approach is the intertrochanteric osteotomy, which is performed as a horizontal cut between the lesser and major trochanter. Cuts closer to the deformity (i.e. in the femoral neck) are a more direct 
approach concerning the correction, but it is recognized that these have a much higher incidence of postoperative femoral head necrosis.

Since Imhäuser and Southwick described the guidelines for intertrochanteric osteotomies, little has changed in the planning of these surgeries $[6,13]$. In general the deformity is diagnosed and the amount of the displacement is measured on biplanar plain radiographs. The measurements give the values for the amount and direction of the reorientation of the proximal part.

The major uncertainties of this method are the poor reproducibility of the positioning for radiographs and the projectional errors [3]. In addition, the reorientation consists of a combination of rotations along different axes. A surgical plan based on biplanar radiographs doesn't address this complex procedure adequately.

The ability to acquire volumetric image data instead of projectional images and digital image post-processing could improve surgical planning. So far the planning of reorienting osteotomies in SCFE does not benefit from those technologies on a routine basis. Some earlier proposed systems and algorithms could be applied to this problem $[1,4,9,10,14]$, but all of them are either missing interactivity or lack the ability to functionally evaluate the simulated procedure.

It was therefore our goal to develop an environment for the simulation of reorienting osteotomies. Such a system should be:

- fast enough for routine clinical use,

- able to simulate an osteotomy,

- able to realign anatomical objects separately and

- able to allow a functional evaluation.

\section{Methods}

We developed a surgical planning environment for reorienting osteotomies in cases of SCFE. Three-dimensional surface models represent the relevant anatomical structures in our approach.

In the first step, the preoperative range of motion based on the bony constraints can be determined by simulating the hip motion. Collision detection is used to control the motion in the hip joint. Our model does not use a fixed and predetermined rotation center.

The next step is the simulation of the osteotomy and the reorientation. Then the surface models are saved in the new position and finally reevaluated by the motion simulation.

In the following paragraphs, the underlying techniques are described in detail.

\subsection{Visualization}

In this study CT volume scans are used to reconstruct three-dimensional surface models. Segmentation of femur and pelvis is done with commonly used intensity thresholding techniques. Operator interaction is needed ensure proper separation of pelvis and femur and to determine the border between femoral head and neck. The latter is equivalent to the growth plate, which appears much darker than calcified bone in CT images.

The segmented files are reconstructed using "The Visualization Toolkit" (VTK) [11] implementation of the marching cubes algorithms [7] to the following objects: pelvis, femoral head and femoral neck with proximal femur (Fig. 1). In addition, the 
position of the knee condyles is represented to check for ante-/retroversion and the rotation of the femur. The surface models typically consist of about 50,000 triangles, after application of a triangle reduction algorithm [12].

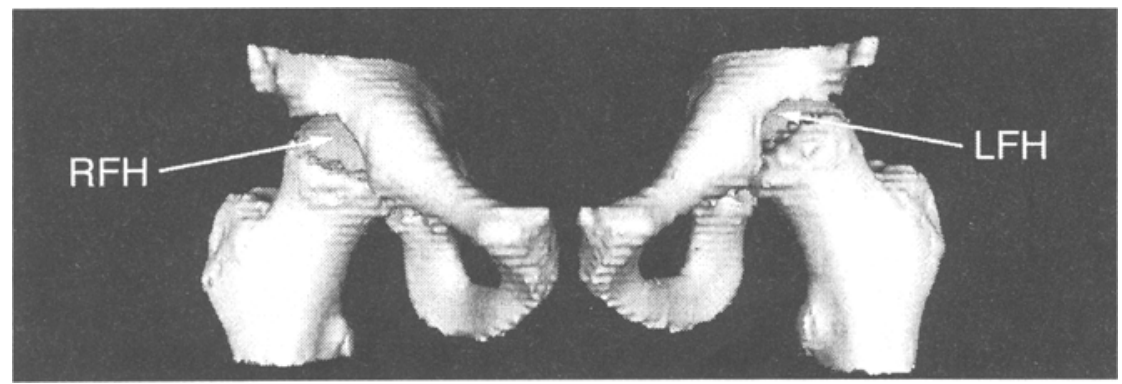

Fig. 1. Frontal view of the surface models of both hips in neutral position. The left hip is affected by SCFE. The left femoral head (LFH) is displaced posterior and slightly inferior. The right femoral head (RFH) shows a normal geometry.

The discrimination between femoral heads and the rest of the femur distinguishes between objects with and without articular cartilage.

The rendering uses the VTK libraries as well [11].

\subsection{Collision Detection}

For collision detection, we used the VCollide library of the University of North Carolina at Chapel Hill [5]. This library checks for interference between triangles of different geometric objects. The objects have to be represented as so-called polygon soups. The objects do not have to be convex and they are not considered to have any special properties. In our case we have convex and concave objects as well.

In a first step, VCollide computes a hierarchical tree-structure of oriented bounding boxes (OBB). Following certain rules, a generated OBB is separated in two areas with nearly the same number of triangles. For each of these areas, a new OBB is generated and so on. This leads to a hierarchical OBB-tree-representation.

OBB's are especially useful in interference tests (aka. collision detection). Only the OBB's are transformed - not the whole object. Only if two OBB's are not disjoint is the next layer of OBB's tested. If leaf nodes are reached in both structures, then all the triangles, which are represented by these nodes, are transformed and tested for interference. This method reduces the amount of transformations as well as the amount of interference tests, and is largely responsible for the interactive performance of our system.

\subsection{Determination of an arbitrary rotation center}

We model flexion and abduction as rotations of the femur. Therefore, we need to estimate an arbitrary center of these rotations for both femora. The positions of these centers relative to the acetabulum will change during motion simulation, as each femur is repositioned to mate its joint surface with the corresponding surface of the acetabulum. 
The femoral head can be approximated as part of a sphere of which we can compute the center. We designated the triangle in the segmented femoral head with the most superior centroid as belonging to the convex surface of the joint.

With these assumptions we can easily classify the convex and concave portions of the surface. The convex portion is designated as the joint surface, and is used for the fully automatic determination of the rotation center. For several points of the convex portion we estimate the normals. On a perfect sphere, these normals would all intersect at the center of rotation. Because our segmentation result is not a perfect sphere, the normals usually do not intersect each other. Therefore, we determine the shortest connection of all these normals; the center of gravity of these connections is then called arbitrary center of rotation.

It is not important for the rotation center to be computed precisely. An inaccurate estimation only reduces the performance of the simulation process by increasing the number of iterations needed to reposition the femora in order to mate joint surfaces.

\subsection{Motion Simulation}

Motion simulation consists of reorienting the femur by small increments while maintaining contact between paired joint surfaces, and checking that no other pairs come into contact. Motion stops when further rotation of the femur under these constraints is impossible.

Since contacts of parts not covered with cartilage are non-physiologic, only our femoral head model is allowed to collide with the pelvis object.

To hold the femur in the acetabulum, we simulate a force to the femur towards the acetabulum. For each step of the simulation process, the femur is forced in this direction as far as possible. If a collision is detected, small steps perpendicular to the direction of the force are performed. For each of these steps, it is checked whether the femur can move further towards the acetabulum. With this iterative scheme, we find the optimal position of the femur.

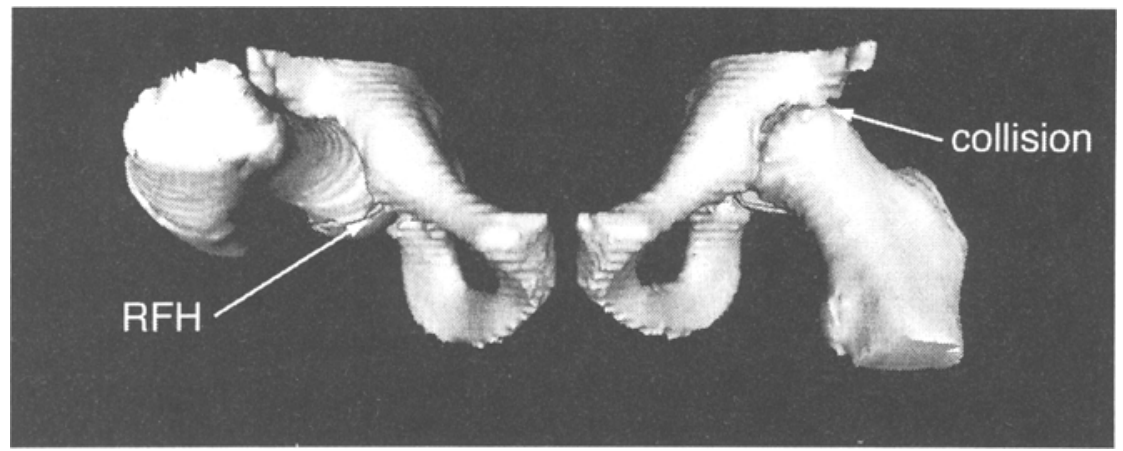

Fig. 2. Frontal view of the surface models of both hips, maximal simulated flexion. The left femur is flexed $29^{\circ}$, the right femur $105^{\circ}$. The location of the bony motion-limiting collision is indicated for the left femur.

After the optimal position is found, the program tries to rotate the femur along a user-defined axis (i.e. for flexion or abduction). This process is performed stepwise as 
well and alternates with the formerly described femur position adjustment. This alternating scheme allows a motion adapted to the individual surface geometry of the joint partners without being committed to an arbitrary rotation center.

For each simulation step, the collision detection algorithm is applied. The simulation process stops as soon as femoral parts other than the head collide with any part of the pelvis (Fig. 2 and 4).

Remark. In order to bias these findings, we have to put the femora in a neutral position before simulation. Most important in this aspect seems to be the femoral rotation. Due to the slippage of the epiphysis the patient keeps the leg in external rotation. The virtual models of the knee condyles enable us to correct for this problem.

\subsection{Osteotomy Simulation}

The osteotomy simulation tool permits the operator to read, view, cut, and reorient segmented surface models. The visualization functions are based on the VTK libraries [11], but it adds the ability to perform boolean operations between different surface models. In the case of osteotomy simulation, it is the boolean subtraction of the cutter model (i.e. virtual saw) from the bone model with interactive performance. At the core of the operation is the calculation of intersections between the edges of one model and the triangles of the other model [8].

The same OBB hierarchy mentioned earlier is used to rapidly identify regions of potential interaction between cutter surface and bone surface [5]. Therefore, topology and transformations are only computed for those sets of triangles that are identified in the OBB hierarchy for potential interaction.

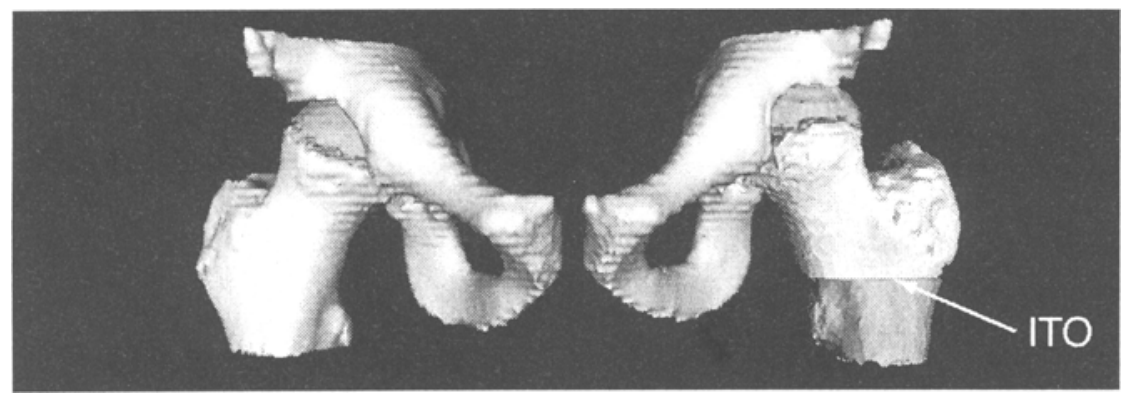

Fig. 3. Frontal view of the surface models of both hips after simulated cutting and repositioning. ITO indicates the location of the intertrochanteral osteotomy. The portion of the femur that is distal to the intertrochanteral cut is shown in a darker shade. The proximal femur was repositioned by $20^{\circ}$ of flexion, $25^{\circ}$ of derotation and $5^{\circ}$ of adduction. Note the increased appearance of symmetry in the placement of the left femoral head towards the acetabula.

The cutter model has mouse-driven interface, which can be used to position and orient the tool with respect to the bone. Once positioned, a button press signals the subtraction of the tool volume from the bone volume. For a simple cut, a single subtraction may be sufficient, but multiple cuts can be made. After the osteotomy, the 
bone model consists of two or more topologically disjoint segments (Fig.3). Each segment is identified and written out as an independently orientable model.

The mouse-driven interface can also be used to reposition one segment with respect to the others. During a surgical simulation, the surgeon can reorient the proximal part interactively. While comparing the relative position of femoral head and acetabulum to the position on the healthy side, he can simulate an arbitrary reorientation (Fig. 3).

The same OBB-tree based collision detection that is used for range of motion analysis is also used to prevent positioning with overlapping volumes. At this point, the user may wish to use some of the measuring tools provided for measuring the angles and distances between features of the model.

Once the desired repositioning is achieved, the segments are reunited into a single model, which can be input to the post-surgical range of motion analysis. If the result of the 'postoperative' finding is not satisfying another reorientation can be simulated.

\section{Results}

\subsection{Illustrative Case}

Figures 1-6 are based on the datasets from a 13-year-old boy with SCFE of his left hip (Fig. 1). By simulating the bone determined range of motion we could assess a maximal flexion of $105^{\circ}$ on the healthy side and $29^{\circ}$ on the affected side (Fig. 2). The measurements for abduction were $76^{\circ}$ and $49^{\circ}$ respectively (Fig. 3 ).

In the next step, an intertrochanteric osteotomy was simulated. The proximal part of the femur was flexed by $20^{\circ}$ about the left-right axis, then derotated by $25^{\circ}$ about the vertical axis, followed by a minor valgisation (adduction) of $5^{\circ}$ about the front-back axis. It is important to realize that the final orientation depends on the order of these rotations.

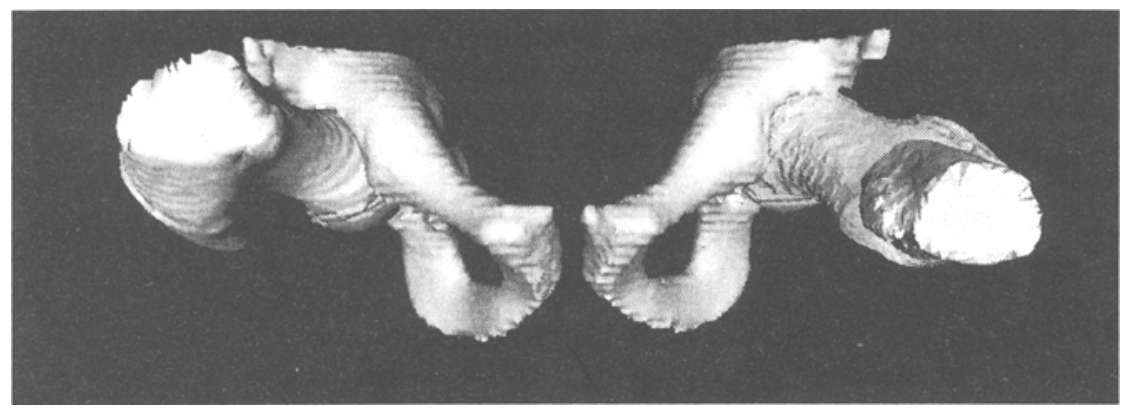

Fig. 4. Frontal view of the surface models of both femora in maximal simulated post-surgical flexion. The left femur is flexed $69^{\circ}$, an improvement of $40^{\circ}$ from the pre-surgical simulation. The right femur is unchanged at $105^{\circ}$.

\subsection{Performance}

We tested our implementation on a SUN ULTRA $30,300 \mathrm{MHz}, 128 \mathrm{MB}$ RAM, with Creator3D graphics acceleration and on a SGI $\mathrm{O}_{2}, \mathrm{R} 10000,180 \mathrm{Mhz}, 128 \mathrm{MB}$ RAM, 
with standard 3D triangle acceleration. Our scenes usually consist of 8 objects with approximately 50,000 triangles. In general, the data size for those models is about one MByte.

Table 1. Performance of our collision detection system on different computer systems.

\begin{tabular}{ccc}
\hline $\begin{array}{c}\text { Computer } \\
\text { System }\end{array}$ & $\begin{array}{c}\text { Preparing } \\
\text { OBB-structure }\end{array}$ & $\begin{array}{c}\text { Collision } \\
\text { detection }\end{array}$ \\
\hline SUN & $3.20 \mathrm{sec}$ & $0.05 \mathrm{sec}$ or $16 \mathrm{fps}$ \\
\hline SGI & $4.77 \mathrm{sec}$ & $0.07 \mathrm{sec}$ or $14 \mathrm{fps}$ \\
\hline
\end{tabular}

For these measurements, the collision detection is performed in real time using a standard UNIX workstation. A full motion to maximal flexion / abduction for both sides takes usually between 10 and 30 seconds.

The osteotomy simulation is performed in real time as well.

\section{Discussion}

We present a system for the preoperative evaluation, simulation of reorienting osteotomies and the 'postoperative' reevaluation in cases of SCFE.

Previously described systems generally applicable to this topic lack some important features. Simple and fast simulation systems are mostly based on surface models. These systems are able to perform osteotomies, but due to their lack of collision detection, they are neither able to simulate the improvement in the range of motion nor to simulate a hip motion without a fixed center of rotation $[1,9]$.

Gerber's approach uses volume rendering to display the motion of the hip joint [4]. This procedure comes at the cost of time. The vast amount of necessary computations makes real time interaction impossible.

Pflesser et al. used a method of ray composition and rendering [10]. This method can display interpenetrating volumes. However, the motion of the hip joint requires a predefined and fixed center of rotation. In addition, this system does not detect and avoid the collision automatically. It needs the evaluation of a user.

For the known problems in surgical simulation systems, we developed a system based on surface models, which we fitted by oriented bounding boxes (OBB). The combination of OBB's and surface models is the basis for the high performance of our system. In contrast to axis-aligned bounding boxes (AABB), OBB's fit the object better [5]. Although the computation of OBB's is more time-consuming than that of AABB's, OBB's do not have to be recomputed if the object is transformed.

Anatomically, our system currently lacks the interaction of ligaments and muscles. Therefore, in some cases, non-physiological degrees of motion could be observed. The addition of ligament modeling to the existing environment is currently in progress. This will make the current system applicable to many other joints and surgical planning problems.

Using the current system, a surgeon can already obtain valuable information for the planning of reorienting osteotomies in cases of SCFE. Instead of estimating the impact of his planned osteotomy to the improvement of range of motion, he can visualize and quantify that impact in a three dimensional environment.

Due to the performance of the system, even on standard workstations, the practicality of the system has a major advantage over earlier programs. In addition, the data preparation can be done in a reasonable amount of time. 
On the whole, the presented system can be used for the planning of reorienting osteotomies in cases of SCFE on a routine clinical basis.

\section{References}

1. Altobelli D, Kikinis R, Mulliken J, Cline H, Lorensen W, and Jolesz F, 1993 Computer-assisted three-dimensional planning in cranofacial surgery. Plast Reconst Surg, 92: 576-585.

2. Boyer DW, Mickelson MR, and Ponseti IV, 1981

Slipped capital femoral epiphysis. Long-term follow-up study of one hundred and twenty-one patients.

J Bone Joint Surg [Am], 63: 85-95.

3. Cohen MS, Gelberman RH, Griffin PP, Kasser JR, Emans JB, and Millis MB

Slipped capital femoral epiphysis: assessment of epiphyseal displacement and angulation.

J Pediatr Orthop, 6: 259-264.

4. Gerber JD, Ney DR, Magid D, and Fishman EK, 1991

Simulated Femoral Repositioning with Three-Dimensional CT.

J Comput Assist Tomogr, 15: 121-125.

5. Gottschalk S, Lin M, and Manocha D, 1996

OBB-Tree: A Hierarchical Structure for Rapid Interference Detection.

Proc. ACM Siggraph '96, 171-180.

6. Imhäuser $\mathrm{G}, 1957$

Zur Pathogenese und Therapie der jugendlichen Hüftkopflösung.

Z Orthop, 88: 3-41.

7. Lorensen W and Cline H, 1987

Marching cubes: A high resolution 3D surface construction algorithm.

Computer Graphics, 21: 163-169.

8. Mantyla M, 1988

An Introduction to Solid Modelling.

Computer Science Press, Rockville, MD.

9. Murphy SB, Kijewski PK, Millis MB, and Harless A, 1989

Simulation of Osteotomy Surgery about the Hip Joint.

Computer Assisted Radiology, Proc. CAR '91, Lemke, H. U. et al. (eds.), SpringerVerlag, Berlin.

10. Pflesser B, Tiede U, and Höhne KH, 1994

Simulating Motion of Anatomical Object with Volume-Based 3D-Visualisation.

Visualization in Biomedical Computing, Proc., R. A. Robb (ed.), SPIE 2359, Rochester, MN, 291-300.

11. Schroeder W, Martin K, and Lorensen W, 1997

The Visualization Toolkit.

2nd ed., Prentice-Hall, New Jersey.

12. Schroeder, W., J. Zarge, and W. Lorensen. 1992.

Decimation of Triangle Meshes.

Computer Graphics 26(2):65-70.

13. Southwick WO, 1967

Osteotomy through the Lesser Trochanter for Slipped Capital Femoral Epiphysis.

J Bone Joint Surg, 49-A: 807-835.

14. Vannier M and Marsh J, 1996

Three-dimensional imaging, surgical planning and image-guided therapy.

Rad Clin North Am, 34: 545-563. 